\title{
Increased Levels of Diadenosine Polyphosphates in Dry Eye
}

\author{
Assumpta Peral, ${ }^{1}$ Gonzalo Carracedo, ${ }^{1}$ M. Carmen Acosta,${ }^{2}$ Juana Gallar, ${ }^{2}$ \\ and Jesús Pintor ${ }^{3}$
}

Purpose. To analyze the levels of the diadenosine polyphosphates $\mathrm{Ap}_{4} \mathrm{~A}$ and $\mathrm{Ap}_{5} \mathrm{~A}$ in tears, in a set of control subjects and in groups of symptomatic and nonsymptomatic persons with dry eye.

Methods. Ninety-seven subjects participated in the study. The subjects were divided into five experimental groups: control subjects; symptomatic patients with normal tear secretion; symptomatic patients with low tear secretion; forced blink; and corneal mechanical stimulation provided by a gas esthesiometer. The Schirmer I test was used to measure and collect tear secretions from each subject. All samples were processed by high pressure liquid chromatography (HPLC) and their $\mathrm{Ap}_{4} \mathrm{~A}$ and $\mathrm{Ap}_{5} \mathrm{~A}$ levels determined.

Results. The levels of $\mathrm{Ap}_{4} \mathrm{~A}$ and $\mathrm{Ap}_{5} \mathrm{~A}$ in tears were greater in all symptomatic patients than in control subjects, especially in symptomatic subjects with low tear secretion. Within the symptomatic subjects with normal tear secretion, significant differences in concentrations of $A p_{4} A$ and $A p_{5} A$ were found between men and women. In the forced blink experiments, concentrations of the $A_{4} A$ and $A p_{5} A$ rose with increasing blink frequency. When the cornea was mechanically stimulated, the levels of $\mathrm{Ap}_{4} \mathrm{~A}$ and $\mathrm{Ap}_{5} \mathrm{~A}$ rose significantly during both moderate and high-flow rate tests.

Conclusions. The increased levels of $A p_{4} A$ and $A p_{5} A$ in tears of patients with dry eye allow these dinucleotides to be used as objective biomarkers in dry eye conditions. (Invest Ophthalmol Vis Sci. 2006;47:4053-4058) DOI:10.1167/iovs.05-0980

$\mathrm{T}$ he tear film constitutes a moist natural barrier that separates the eye from the external media. This consistent film is formed mainly by a triplet of aqueous, mucous, and lipid layers that provide the necessary equilibrium for maintaining the health of the ocular surface. ${ }^{1}$ The principal functions of this film are: (1) to keep the ocular surface wet and well lubricated; (2) to transfer the nutritional elements to the cornea, (3) to eliminate foreign matter and cellular debris gener-

From the ${ }^{1}$ Departamento de Óptica II (Optometría y Visión), Escuela Universitaria de Óptica, Universidad Complutense de Madrid, Madrid, Spain; ${ }^{2}$ Instituto de Neurociencias de Alicante, Universidad Miguel Hernández-CSIC, Sant Joan d'Alacant, Spain; and ${ }^{3}$ Departamento de Bioquímica y Biología Molecular IV, Escuela Universitaria de Óptica, Universidad Complutense de Madrid, Madrid, Spain.

Supported by research grants CENIT (Nanofarma) from the Ministerio de Industria y Comercio and SAF2005-07277 from the Ministerio de Educación y Ciencia, Spain.

Submitted for publication July 27, 2005; revised December 15 , 2005, and April 19, 2006; accepted July 24, 2006

Disclosure: A. Peral, None; G. Carracedo, None; M.C. Acosta, None; J. Gallar, None; J. Pintor, None

The publication costs of this article were defrayed in part by page charge payment. This article must therefore be marked "advertisement" in accordance with 18 U.S.C. $\$ 1734$ solely to indicate this fact.

Corresponding author: Assumpta Peral, Dept. de Óptica II (Optometría y Visión), Escuela Universitaria de Óptica, Universidad Complutense de Madrid, Avda Arcos de Jalón s/n, 28037 Madrid, Spain; assumpta@opt.ucm.es. ated on the ocular surface by the tear flow and the blink process; and (4) to act as the first line of defense against ocular surface infections. ${ }^{2}$

The balance between the composite layers of the tear film is critical, and when altered it can give rise to complications on the ocular surface. These complications are widely known as dry eye-related problems, the severity of which depends on the level of imbalance. Two causes of this disequilibrium are a deficiency in tear secretion caused by Sjögren's syndrome, or tear film instability due to the wearing of contact lenses. Signs and symptoms of these complications can vary from patient to patient, often with little or no correlation between them. Patients may present with low tear secretion volume, ocular signs, and no ocular symptoms or with normal tear volume and ocular symptoms of dryness. This poor correlation is well known, having been frequently reported in the literature. ${ }^{3-5}$ This ambiguity in etiology and pathophysiology ${ }^{6}$ can make a precise diagnosis difficult.

Today, there are several questionnaires and tests designed to evaluate patients' signs and symptoms. Among these tests, the National Eye Institute/Industry Dry Eye Workshop ${ }^{7}$ proposed the combined use of a validated questionnaire regarding symptoms, a test to evaluate ocular surface damage, measurement of tear instability, and the demonstration of tear hyperosmolarity.

More recently, the second National Eye Institute/Industry Workshop on Clinical Trials has given rise to new criteria for subjective and objective testing for dry eye, and several tests, both invasive and noninvasive, are now emerging. ${ }^{8}$ These tests are designed to identify and analyze biomarker profiles in tears to detect clearly whether an individual has dry eye. The identification of a stable molecule present in tears whose concentration changes with different dry eye conditions, would make diagnosis, follow-up, and the assessment of dry eye treatments both easier and more precise.

Pintor et al. ${ }^{9}$ have described the presence of a new family of compounds in the tear film, the diadenosine polyphosphates. These compounds, are naturally occurring dinucleotides, exhibiting both intracellular and extracellular physiological effects $^{10,11}$ Formed by two adenosine molecules joined by a variable phosphate chain, they are abbreviated as $\operatorname{Ap}_{\mathrm{n}} \mathrm{A}(n=$ 2-7). Although the activity of these nucleotides in ocular tissues has not been fully investigated, it is known that they act through $\mathrm{P}_{2}$ receptors to modulate intraocular pressure in rabbits $^{12} ; \mathrm{Ap}_{4} \mathrm{~A}$ and UTP improve the rate of wound healing in the cornea of New Zealand White rabbits ${ }^{13} ; \mathrm{Ap}_{4} \mathrm{~A}, \mathrm{Ap}_{5} \mathrm{~A}$, and $A \mathrm{p}_{6} \mathrm{~A}$, can stimulate tear secretion after single-dose topical application in rabbits. ${ }^{14}$

In this study, the levels of diadenosine polyphosphates in tear secretions were examined in a group of control subjects without any symptom of ocular dryness and with a normal volume of tear secretion, as well as two groups of dry eye symptomatic subjects, one with low tear secretion volume and another with normal tear volume. Secretion of the dinucleotides was also examined in forced blink experiments and after moderate and strong corneal mechanical stimulation using a gas esthesiometer. Analysis of the resultant data suggests that 
these substances, in particular $\mathrm{Ap}_{4} \mathrm{~A}$, can act as a tear film molecular marker for dry eye states. Preliminary results have been reported in abstract form (Peral A, et al. IOVS 2003;44: ARVO E-Abstract 2494).

\section{MeTHODS}

\section{Subjects}

Ninety-seven subjects of both sexes ( 27 men and 70 women, of age ranging from 20 to 36 years with a mean age of $27 \pm 1$ years) participated voluntarily in the study. The research was in compliance with the tenets of the Declaration of Helsinki. The subjects signed an informed consent and were free to interrupt the session at any time. The McMonnies ${ }^{15}$ test was performed to detect possible symptoms of ocular dryness.

As identified by the McMonnies test, all nonsymptomatic subjects $(n=51)$, had a Schirmer I test ${ }^{16}$ result equal to or greater than $10 \mathrm{~mm}$. This population was then divided into three groups: those who would participate in the forced-blink experiment $(n=8)$, those who would take part in the esthesiometer measurements $(n=6)$, and a control group $(n=37)$.

Symptomatic subjects $(n=46)$, again as identified by the McMonnies test, were divided into two groups: symptomatic subjects with normal tear secretion $(n=34)$, those who presented symptoms of ocular dryness and had a Schirmer I test result $\geq 10 \mathrm{~mm}$; symptomatic subjects with low tear secretion $(n=12)$, those who exhibited symptoms but whose tear secretion measured with the Schirmer I test was $\leq 5 \mathrm{~mm}$.

The distribution of subjects and McMonnies scores for the different groups are summarized in Table 1.

\section{Trials}

Tear Collection. Tear secretion was measured in all groups with the Schirmer I test. In control group and symptomatic subjects, the Schirmer strip was located on the temporal tarsal conjunctiva of the lower lid for 5 minutes with the eyes closed. Secretion was measured without any kind of stimulation.

Forced-Blink Experiment. Schirmer trips were located at the temporal tarsal conjunctiva of the lower lid of eight nonsymptomatic subjects with normal tear secretion. The patients were instructed to blink at $0,12,30$, and 60 blinks per minute during 5 minutes.

Esthesiometry Experiment. Schirmer strips were located at the temporal tarsal conjunctiva of the lower lid of six nonsymptomatic subjects with normal tear secretion for 5 minutes after the corneal stimulation. The cornea was mechanically stimulated with a gas esthesiometer. ${ }^{17}$ Three gas pulses, each lasting 3 seconds, were applied sequentially (interval, $<0.5$ seconds) to the center of the cornea. Two flow rates of mechanical stimulation were applied: moderate (170 $\mathrm{mL} / \mathrm{min})$ and high $(260 \mathrm{~mL} / \mathrm{min})$ flow. ${ }^{18}$ Subjects were asked to avoid blinking during the application of the three gas pulses.

In all groups, the volume of tears, measured as millimeters of moistened Schirmer strip, was recorded and the strips placed in tubes (Eppendorf, Fremont, CA) containing $500 \mu \mathrm{L}$ of ultrapure water. The tubes were then frozen until HPLC analysis was performed.

\section{Sample Processing and HPLC Analysis}

Schirmer strips were collected, placed in Eppendorf tubes containing $500 \mu \mathrm{L}$ of ultrapure water, and strongly vortexed for 5 minutes. The strips were carefully rinsed and the liquid in the tube was heated in a $100^{\circ} \mathrm{C}$ bath for 20 minutes to precipitate proteins. The tubes were centrifuged at $4000 \mathrm{rpm}$ for 30 minutes to form a pellet of the proteins. Diadenosine polyphosphates are resistant to this treatment, as demonstrated by Pintor et al. ${ }^{19}$ The supernatants were chromatographed (SEP-PAK Accell QMA cartridges; Waters, Milford, MA). ${ }^{20}$ Briefly, 250 $\mu \mathrm{L}$ of the supernatant was passed through the cartridges which had been equilibrated with $3 \mathrm{~mL}$ of ultrapure water. The elution of the nucleotides and dinucleotides was performed by applying $1 \mathrm{~mL}$ of a solution containing $0.2 \mathrm{M} \mathrm{KCl}$ and $0.1 \mathrm{M} \mathrm{HCl}$, and the samples were neutralized with $\mathrm{KOH}$. The eluents were then injected at a volume of 10 to100 $\mu \mathrm{L}$ into the HPLC for analysis.

Determination and quantification of diadenosine polyphosphates were performed by HPLC. The chromatographic system consisted of a isocratic HPLC pump (model 1515; Waters), a dual absorbance detector (2487; Waters), and an injector (Reodyne, Rhonert Park, CA), all managed by the software (Breeze) and a column from Waters $(15 \mathrm{~cm}$ length, $0.4 \mathrm{~cm}$ diameter; Novapack C18).

The system was equilibrated overnight with the following mobile phase: $0.1 \mathrm{M} \mathrm{KH}_{2} \mathrm{PO}_{4}, 2 \mathrm{mM}$ tetrabutyl ammonium, and $17 \%$ acetonitrile ( $\mathrm{pH} 7.5) .{ }^{19}$ Detection was monitored at a $260-\mathrm{nm}$ wavelength, and all the peaks identified as putative dinucleotides were taken for phosphodiesterase treatment. Phosphodiesterase from Crotalus durissus, from Sigma-Aldrich (St. Louis, MO; EC 3.1.15.1.) at a concentration of $0.3 \mathrm{U} / \mathrm{mL}$ was incubated for 10 minutes with the corresponding putative dinucleotide. The digestion products were analyzed by HPLC. Peaks were transformed into concentrations by means of external commercial diadenosine polyphosphates standards of known concentrations.

\section{Statistical Analysis}

The data are presented as the mean \pm SD of results of the experiments. Data were analyzed with Student's $t$-test, and significance was set at $P<0.05$.

\section{Results}

\section{$\mathrm{Ap}_{4} \mathrm{~A}$ and $A p_{5} \mathrm{~A}$ Levels for the Control Group and Symptomatic Subjects}

Pintor et al. ${ }^{9}$ identified the presence of diadenosine polyphosphates in human tears; however, little is known about the levels of concentration in the tears of individuals presenting with ocular dryness. The levels of these substances in both normal and pathologic eyes were therefore investigated in the present study.

Two peaks were tentatively identified as $A p_{4} A$ and $A p_{5} A$, according to the HPLC retention times (Fig. 1A). To confirm that these peaks corresponded to $A p_{4} A$ and $A p_{5} A$, they were individually collected and treated with phosphodiesterase from Crotalus durissus (Figs. 1B, 1C). The treatment of the putative $\mathrm{Ap}_{4} \mathrm{~A}$ produced two peaks after the enzyme action, identified

Table 1. Distribution of McMonnies Scores in the Different Groups

\begin{tabular}{|c|c|c|c|c|c|}
\hline & \multirow[b]{2}{*}{ Control } & \multicolumn{2}{|c|}{ Nonsymptomatic $(n=51)$} & \multicolumn{2}{|c|}{ Symptomatic $(n=46)$} \\
\hline & & Forced Blink & $\begin{array}{l}\text { Esthesiometer } \\
\text { Measurements }\end{array}$ & $\begin{array}{l}\text { Normal Tear } \\
\text { Secretion }\end{array}$ & $\begin{array}{l}\text { Low Tear } \\
\text { Secretion }\end{array}$ \\
\hline Number & 37 & 8 & 6 & 34 & 12 \\
\hline Women/men & $24 / 13$ & $6 / 2$ & $4 / 2$ & $24 / 10$ & $12 / 0$ \\
\hline Scores & $5.1 \pm 2.2$ & $5.1 \pm 2.4$ & $8.1 \pm 2.1$ & $13.3 \pm 2.1$ & $12.8 \pm 1.6$ \\
\hline
\end{tabular}




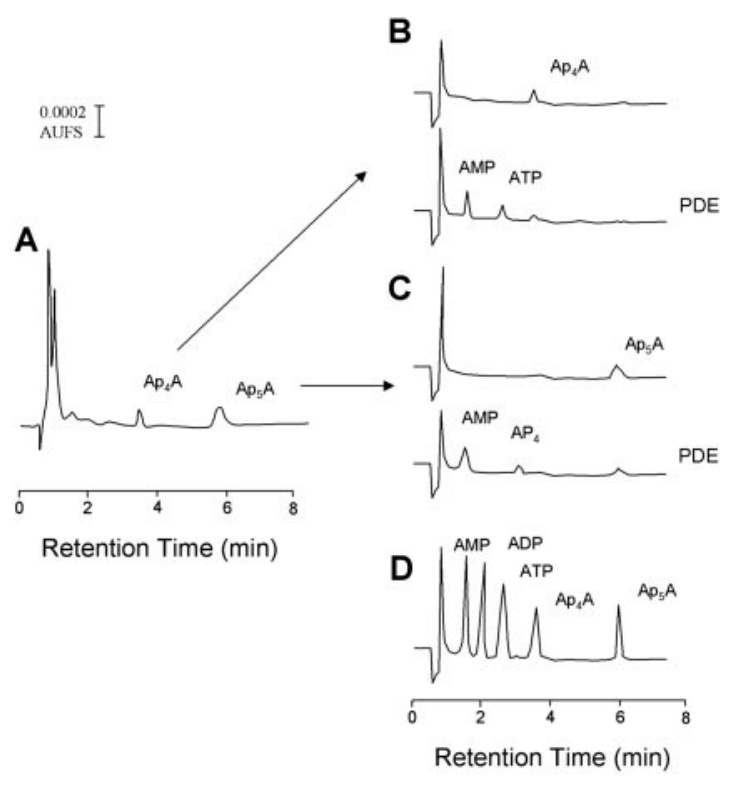

Figure 1. Diadenosine polyphosphate detection in tears. (A) Chromatographic profile of a sample obtained and processed, showing the presence of two peaks identified as $\mathrm{Ap}_{4} \mathrm{~A}$ and $\mathrm{Ap}_{5} \mathrm{~A}$. (B) Rechromatography of the $\mathrm{Ap}_{4} \mathrm{~A}$ peak shown in (A), after treatment with phosphodiesterase. The reduction in the dinucleotide peak is concomitant with the appearance of the hydrolytic products AMP and ATP. (C) Rechromatography of the $\mathrm{Ap}_{5} \mathrm{~A}$ peak shown in (A) after treatment with phosphodiesterase. The reduction in the dinucleotide peak is concomitant with the appearance of the hydrolytic products AMP and $\mathrm{Ap}_{4}$. (D) Standard injection of commercial adenine mono- and dinucleotides.

as AMP and ATP, whereas the putative $\mathrm{Ap}_{5} \mathrm{~A}$ yielded AMP and adenosine tetraphosphate $\left(\mathrm{Ap}_{4}\right)$. These results indicate that the two peaks did indeed correspond to $A p_{4} A$ and $A p_{5} A$. Comparison of the strength of these peaks with known standards permitted quantification of the concentration of the two dinucleotides in the samples (Fig. 1D). In the control group, the concentrations observed for diadenosine tetraphosphate and diadenosine pentaphosphates were $0.107 \pm 0.291$ and $0.036 \pm 0.030 \mu \mathrm{M}$, respectively ( $n=37$; Fig. 2 ).

Relative to the control group, the symptomatic group with low tear secretion showed a marked increase in diadenosine polyphosphate concentrations, statistically to a $95 \%$ or greater confidence level (Fig. 2), their concentrations being $10.68 \pm$ $1.87 \mu \mathrm{M}$ for $\mathrm{Ap}_{4} \mathrm{~A}$ and $12.45 \pm 0.290 \mu \mathrm{M}$ for $\mathrm{Ap}_{5} \mathrm{~A}(n=12)$. The concentrations of these dinucleotides in the symptomatic subjects with normal tear secretion, exhibited similar, though less marked, variations in the dinucleotide levels (Fig. 2). The concentrations of $\mathrm{Ap}_{4} \mathrm{~A}$ and $\mathrm{Ap}_{5} \mathrm{~A}$ in these cases were $0.590 \pm$ 0.330 and $0.058 \pm 0.017 \mu \mathrm{M}$, respectively $(n=34)$.

A potentially significant observation was made when the sex of the subject was factored in to these results. Whereas the control group exhibited no significant variance in concentration with sex, the sample of symptomatic women with normal tear secretion appeared to present higher levels of diadenosine polyphosphates than did that of men. Symptomatic women presented $\mathrm{Ap}_{4} \mathrm{~A}$ levels of $0.796 \pm 0.330 \mu \mathrm{M}(P=0.001)$, whereas symptomatic men exhibited concentrations of $0.384 \pm 0.172 \mu \mathrm{M}$ for the same dinucleotide. Concerning the levels of $\mathrm{Ap}_{5} \mathrm{~A}$, symptomatic women presented concentrations of $0.069 \pm 0.031 \mu \mathrm{M}$ and symptomatic men of $0.047 \pm 0.015$ $\mu \mathrm{M}(P=0.037)$. It was not possible to repeat this observation for the low tear volumes, however, because all patients in this group were female. The potential divergence gives rise to the possibility that there may be a hormonal factor involved, which may bear further investigation. These mentioned divergences open the possibility of performing future studies to see whether hormonal factors could be responsible for such variability.

\section{$A p_{4} A$ and $A p_{5} A$ Levels and Forced Blinking}

Tsubota et al. ${ }^{21}$ reported that patients with dry eye can increase their blink rate to compensate for tear instability. Accordingly, in this study, the effect of forced blinking on levels of diadenosine polyphosphates in the tear film was examined. Eight subjects with no symptoms of ocular dryness and a normal volume of tear secretion participated in the experiment. Variations in $\mathrm{Ap}_{4} \mathrm{~A}$ and $\mathrm{Ap}_{5} \mathrm{~A}$ concentrations in their tears were investigated for four different blink frequencies: 0 , 12,30 , and 60 blinks per minute, with 0 blinks per minute (eyelids closed) being considered the control for this experiment.

The chromatographic analysis revealed substantial changes in the concentrations of $A p_{4} A$ and $A p_{5} A$ with differing blink frequency (Fig. 3A). When these peaks were transformed into concentrations, the levels of $\mathrm{Ap}_{4} \mathrm{~A}$ rose from $0.109 \pm 0.081 \mu \mathrm{M}$ for $0 \mathrm{blinks} / \mathrm{min}$ to $2.853 \pm 0.394 \mu \mathrm{M}$ at $60 \mathrm{blinks} / \mathrm{min}$ (Fig. $3 B$ ). An almost identical trend was observed for $A p_{5} A$, with concentrations rising from $0.041 \pm 0.050 \mu \mathrm{M}(0 \mathrm{blinks} / \mathrm{min})$ to $2.091 \pm 0.065 \mu \mathrm{M}$ at $60 \mathrm{blinks} / \mathrm{min}$ (Fig. 3B). It was possible to observe a gradual increase in the dinucleotide concentration concomitant with the increase in the blink frequency.

The augmented concentrations of $A p_{4} A$ and $A p_{5} A$ for the different blinking frequencies paralleled an increase in tear secretion. These results are shown in Figure 4 in which an increase in the Schirmer scores occurred with the increase in frequency of the forced blink.

\section{$\mathrm{Ap}_{4} \mathrm{~A}$ and $\mathrm{Ap}_{5} \mathrm{~A}$ Levels for the Esthesiometry Experiments}

The forced blink experiments demonstrated a clear increase in the tear concentrations of $\mathrm{Ap}_{4} \mathrm{~A}$ and $\mathrm{Ap}_{5} \mathrm{~A}$ with increasing blink frequency. This increase could be due to the shear stress produced by the eyelids on the corneal surface. Srinivas et al. ${ }^{22}$ described how the mechanical action of the eyelids on the ocular surface can provoke a shear stress at the corneal epithelial cells and subsequently a release of intracellular substances to the tears including nucleotides and dinucleotides. ${ }^{22}$ One question that arises is whether the release of nucleotides

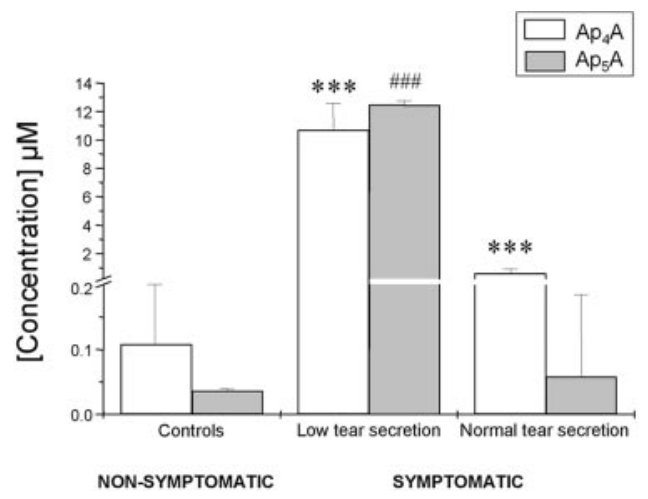

Figure 2. Concentrations of diadenosine polyphosphates in tears in normal and dry eye conditions. Individuals with no symptoms and normal tear secretion (control subjects) were compared with symptomatic patients with either low or normal tear secretion. In all the cases, the dinucleotide concentrations were higher in the symptomatic group than in the control group. In the group of symptomatic normal tear secretors, $A p_{4} A$ but not $A p_{5} A$, was significantly higher than in the control group. Data from both eyes were pooled and are expressed as the mean $\pm \mathrm{SD}$. ${ }^{* * *} P$ and $\# \# P<0.0001$; paired $t$-test. 


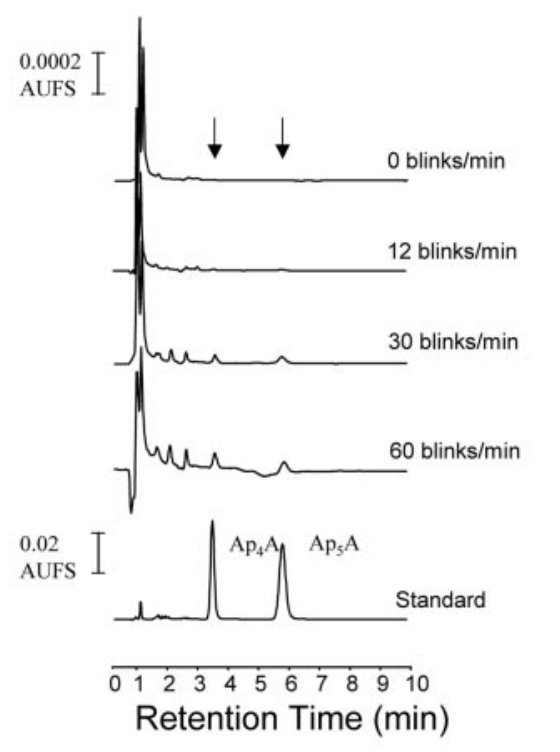

A

B

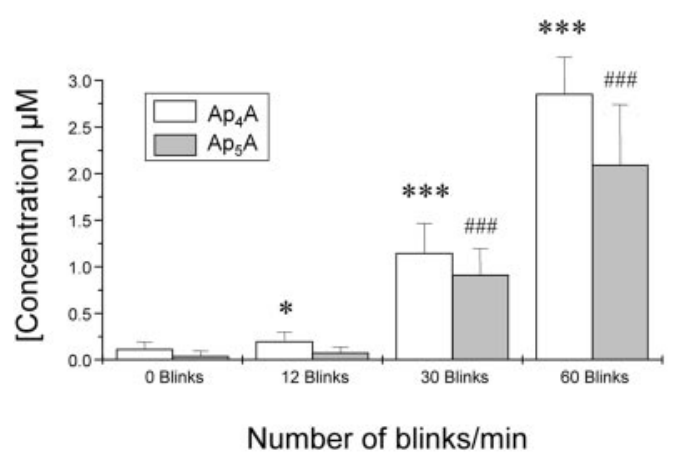

FiguRE 3. Levels of diadenosine polyphosphates after different blinking frequencies. (A) HPLC eluting profiles of tear samples after 0, 12, 30 , and 60 blinks per minute. An increase in the concentration of $\mathrm{Ap}_{4} \mathrm{~A}$ and $\mathrm{Ap}_{5} \mathrm{~A}$ was observed with the increase in the blinking frequency. (B) Quantification of the diadenosine polyphosphate levels in individuals blinking at different frequencies. As occurred in (A), there was a gradual increase in the concentration of $A_{4} A$ and $A p_{5} A$ with the frequency of blinking. Data from both eyes were pooled and are expressed as the mean $\pm \mathrm{SD}$. ${ }^{* * *} P$ and $\# \# P<0.0001$ and ${ }^{*} P<0.05$; paired $t$-test.

is simply a product of this shear stress, or whether it is triggered by mechanical stimulation of corneal nerve terminals. To investigate this, a gas esthesiometer was used to reproduce the mechanical stimulation.

Six subjects participated in this part of the study, all showing a normal volume of tear secretion and none presented symptoms of ocular dryness according to the McMonnies test. Tears were collected after application of the mechanical stimuli, and the diadenosine polyphosphates were isolated and quantified by HPLC.

The concentrations of diadenosine polyphosphates were found to be statistically independent ( $95 \%$ confidence) of the intensity of the mechanical stimulus applied (Fig. 5). Both moderate $(170 \mathrm{~mL} / \mathrm{min})$ and high $(260 \mathrm{~mL} / \mathrm{min})$ mechanical stimulation of the corneal nerves, resulted in a significantly greater amount of dinucleotides than that in the control subjects $(n=6)$. The moderate mechanical stimulus produced tear concentrations of $0.170 \pm 0.012$ and $0.058 \pm 0.073 \mu \mathrm{M}$ for $A p_{4} A$ and $A p_{5} A$, respectively. The $A p_{4} A$ and $A p_{5} A$ concentration for the high mechanical stimulus were $0.163 \pm 0.048$

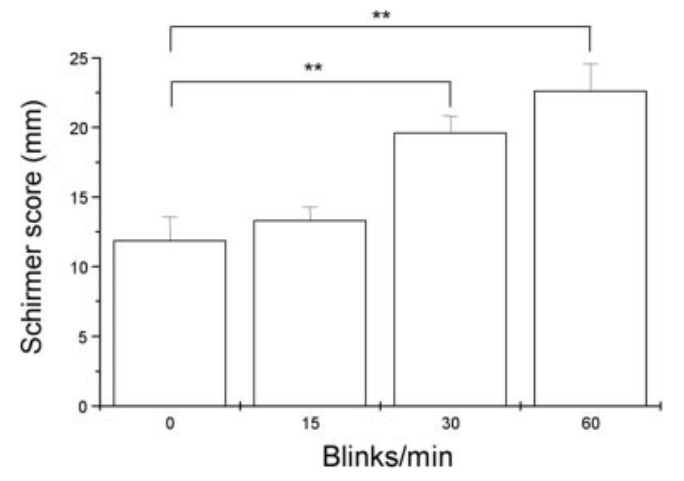

FIGURE 4. Volume of tear secretion measured by Schirmer I test for the different frequencies (bpm) of blinking. The Schirmer test results increased with the increase in the blink rate. Data from both eyes were pooled and are expressed as the mean $\pm \mathrm{SD} .{ }^{* *} P<0.0003$ for $30 \mathrm{bpm}$ and $60 \mathrm{bpm}$, paired $t$-test.

and $0.065 \pm 0.007 \mu \mathrm{M}$, respectively (Fig. 5). Even the moderate mechanical stimulus, which may be of the same intensity as provoked by the eyelids during the blinking process and not strong enough to stimulate the majority of corneal polymodal and mechanonociceptors, resulted in increased concentrations of diadenosine polyphosphates in tears.

\section{Discussion}

The results show that patients with symptomatic dry eye with a reduced tear secretion have greatly elevated levels of the adenosine dinucleotides $\mathrm{Ap}_{4} \mathrm{~A}$ and $\mathrm{Ap}_{5} \mathrm{~A}$ in their tears, being increased 100- and 345-fold for $A_{p_{4}} A$ and $A p_{5} A$, respectively. Symptomatic patients with normal tear secretion also demonstrated an increase in diadenosine polyphosphate concentrations, although not as marked as those with low tear production. These concentrations of $A_{4} A$ and $A p_{5} A$ were increased 5and 1.5-fold, respectively.

Furthermore, a difference between the sexes was evident. Symptomatic women with normal tear secretion presented higher values of diadenosine polyphosphates than did similar men. However, there was no significant difference between the sexes in the control group. This result suggests that the sex of a patient should be taken into account as well as the measure of diadenosine levels in tears, in a specific test to determine borderline dry eye disease.

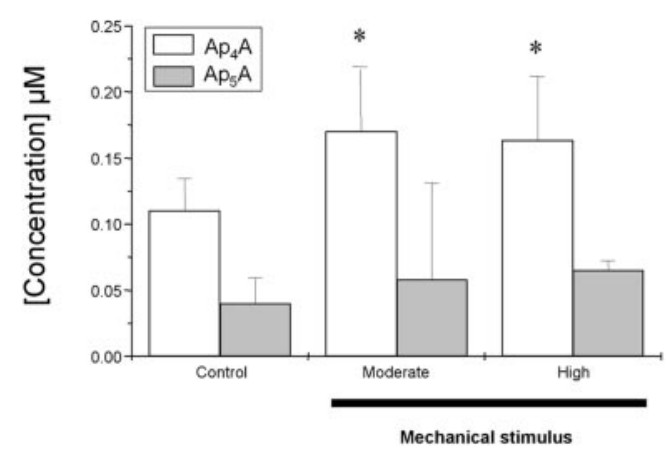

Figure 5. Effect of mechanical stimulation of the cornea with a gas esthesiometer on diadenosine polyphosphate levels in tears. Diadenosine polyphosphates increased in concentration in tears after both moderate and high corneal mechanical stimulation when compared with the control. Data from both eyes were pooled and are expressed as the mean $\pm \mathrm{SD} .{ }^{*} P<0.05$, paired $t$-test. 
Because it has been shown that $A_{p_{4}} \mathrm{~A}$ and $A p_{5} \mathrm{~A}$ stimulate tear secretion, ${ }^{14}$ it was unexpected to see patients with low tear secretion having increased concentrations of these dinucleotides. It appears that although $\mathrm{Ap}_{4} \mathrm{~A}$ and $\mathrm{Ap}_{5} \mathrm{~A}$ are plentiful, they are not able to stimulate lacrimation, and it could be that in those patients, dry eye is a consequence of a lacrimal gland malfunction. The mechanism of action of the dinucleotides is not fully understood. Possibly, they increase tear production by stimulating corneal and conjunctival water and electrolyte production, by causing vasodilatation of conjunctival blood vessels, or by inducing conjunctival goblet cell secretion or increasing meibomian gland secretion. In aqueousdeficiency dry eye, one or more of these processes could be defective, and thus tear volume could be decreased even in the presence of increased levels of $A p_{4} A$ and $A p_{5} A$.

In the symptomatic normal tear secretion dry eye group, tear composition, rather than volume, could be altered causing the symptoms. Dinucleotides present in tears could be stimulating tear secretion, and although the tears are defective, they are produced in normal amounts because of the increased levels of $A p_{4} A$ and $A p_{5} A$. More work is needed to clarify this point.

The mechanism by which diadenosine polyphosphates and other nucleotides such as ATP enter the extracellular fluid has not yet been identified. It is possible, as occurs in the central nervous system, that nucleotides are liberated from nerve terminals, but there is evidence that nucleotides can be transported out of cells. Epithelial cells, ${ }^{23,24}$ and in particular ocular epithelial cells, ${ }^{25}$ use different transport mechanisms as a regulated procedure for nucleotide release. The ATP binding cassette (ABC) transporter, the cystic fibrosis transmembrane conductance regulator (CFTR), or glycoprotein $\mathrm{P}$ have been proposed as elements involved in the release of nucleotides. $^{26-28}$ Gomes et al. ${ }^{29}$ have described that ATP leaves corneal endothelial cells by means of connexin hemichannels when these cells are stimulated mechanically. It would therefore not be surprising that a mechanism of release takes place, although experiments to test this are outside the scope of the present study.

The forced blinking experiments replicate a natural shear stress, and the elevated levels of dinucleotides as a result of increased frequency of blinking are indicative of mechanically stimulated release. It is known that ocular surface conditions can affect the pattern of blinking and that patients with dry eye-related problems can increase their blink rate to compensate for tear instability or deficiency. Tsubota et al. ${ }^{21}$ described a blink rate of 34 blinks per minute in subjects with dry eye, compared with a normal blinking rate of 10 to 15 blinks per minute, which has been described as an essential, involuntary action for the protection of the ocular surface. ${ }^{30,31}$

The elevated levels of dinucleotides in patients with dry eye is compatible with an increased rate of blinking. Patients with dry eye and low tear secretion had values of $A p_{4} A$ and $A p_{5} A$ of 1.14 and $0.91 \mu \mathrm{M}$ respectively, which were comparable to levels recorded in normal subjects forced to blink at 30 per minute. This suggests that an increase in dry eye symptoms followed by an increase of the blinking rate could also produce an enhancement of the $A_{4} A$ and $A p_{5} A$ tear concentrations, and indicate that the appearance of these dinucleotides depends only on the rate of mechanical stimulation, and seems to be independent of tear secretion.

The relationship between the amounts of diadenosine polyphosphates and the blink rate, probably due to the friction of the eyelids on the ocular surface, seems to be clear. The gas esthesiometer ${ }^{17}$ permitted us to apply mechanical stimuli with air at different intensities, close or over the stimulation threshold of corneal nerves. This reveals whether these compounds are released as a consequence of mechanical corneal nerve stimulation.

Acosta et al. ${ }^{18}$ described that only strong stimulation (high mechanical intensity, chemicals, or severe cold) of corneal nerves increases tear secretion, which explains that chiefly stimulation of polymodal corneal sensory nerves evokes reflex tear secretion. Although both moderate and high mechanical stimuli stimulate corneal nerves, high-intensity stimuli recruits a larger population of nerves. ${ }^{32}$ The esthesiometer experiments performed showed a small but significant increase in diadenosine polyphosphate levels after both moderate and strong mechanical stimuli. The lack of difference between the two mechanical stimuli indicates that at least a part of the dinucleotide release is independent of neural stimulation and should respond to the effect of the mechanical force applied to the corneal epithelium. Acosta et al. ${ }^{18}$ reported that a strong stimulus increases tear secretion and also evokes a sensation of irritation that may induce a reflex tear secretion and blinking. This sensation appears more frequently in patients with dry eye and therefore the frequency of blinking is increased. ${ }^{21}$ It is apparent that the blinking process provides ocular protection and contributes to spreading the tear film over the ocular surface. If there is a deficient or unstable tear film, the evaporation rate as well as the necessity of blinking increase. The increase in blinking can induce a shear stress on the ocular surface and, moreover, this fact can lead to raised levels of nucleotides in tears. It has been suggested that these substances, $A p_{4} A$ and $A p_{5} A$, have a role in the stimulation of tear secretion, ${ }^{14}$ and it appears that they are naturally released to try to increase the tear volume/quality stimulating $\mathrm{P}_{2} \mathrm{Y}_{2}$ receptors present in meibomian and accessory glands. ${ }^{33}$ Moreover, because these substances are in any case increased in dry eye, we would like to suggest that these dinucleotides may be used as markers for dry eye conditions.

In summary, the levels of diadenosine polyphosphates in tears were analyzed in different groups of patients with dry eye. The group of symptomatic subjects with low tear secretion as well as the symptomatic subjects with normal tear secretion presented higher levels of diadenosine polyphosphates in tears than did the control subjects. In addition, significant differences were found between men and women and in this symptomatic group, the levels being higher in women. Besides symptomatology, other possible aspects related to dry eye, such as blinking frequency and corneal sensitivity, have been taken into account. While the dinucleotide levels were significantly increased concomitantly with the increase in the blink rate, the contribution from the corneal nerve stimulation was not so important.

These stable molecules could be an objective parameter for scoring the severity of dry eye, the follow-up of the disease, and to determine the efficacy of treatment.

\section{Acknowledgments}

The authors thank Tansy Donovan, Encarna Vives and Charles H. V. Hoyle for help in preparing the manuscript.

\section{References}

1. Records RE. Tear film. In: Records RE, ed. Physiology of the Eye and Visual System. Hagerstown, MD: Harper \& Row, 1979:47-67.

2. Dilly PN. Structure and function of the tear film. Adv Exp Med Biol. 1994;350:239-247.

3. Nichols KK, Nichols JJ, Mitchell GL. The lack of association between signs and symptoms in patients with dry eye disease. Cornea. 2004;23:762-770.

4. McCarty CA, Bansal AK, Livingstone PM, Stanislavsky YL, Taylor HR. The epidemiology of dry eye in Melbourne, Australia. Opbthalmology. 1998;105:1114-1119. 
5. Schein OD, Tielsch JM, Munoz B, Bandeen-Roche K, West S. Relationship between signs and symptoms of dry eye in the elderly: a population-based perspective. Opbthalmology. 1997; 104:1395-1401.

6. Lemp MA. Epidemiology and classification of dry eye. Adv Exp Med Biol. 1998;438:791-803.

7. Lemp MA. Report of the National Eye Institute/Industry workshop on Clinical Trials in Dry Eyes. CLAO J. 1995;21:221-232.

8. Grus FH, Podust VN, Bruns K, et al. SELDI-TOF-MS ProteinChip array profiling of tears from patients with dry eye. Invest Ophthalmol Vis Sci. 2005;46:863-876.

9. Pintor J, Carracedo G, Alonso MC, Bautista A, Peral A. Presence of diadenosine polyphosphates in human tears. Pflügers Arch Eur J Physiol. 2002;443:432-436.

10. McLennan AG. Dinucleoside polyphosphates: friends or foe? Pharmacol Ther. 2000;87:73-89.

11. Hoyle CHV, Hilderman R, Pintor JJ, Schlüter H, King BF. Diadenosine polyphosphates as extracellular signal molecules Drug Dev Res. 2002;52:260-273.

12. Pintor J, Peral A, Peláez T, Martín S, Hoyle CHV. Presence of diadenosine polyphosphates in the aqueous humour: their effect on intraocular pressure. J Pharmacol Exp Ther. 2002;304:342348 .

13. Pintor J, Bautista A, Carracedo G, Peral A. UTP and diadenosine tetraphosphate accelerate wound healing in the rabbit cornea. Ophthalmic Physiol Opt. 2004;24:186-193.

14. Pintor J, Peral A, Hoyle CHV, et al. Effects of diadenosine polyphosphates on tear secretion in New Zealand white rabbits. J Pharmacol Exp Ther. 2002;300:1-7.

15. McMonnies CW, Ho A, Wakefield D. Optimum dry eye classification using questionnaire responses. Adv Exp Med Biol. 1998;438: 835-838.

16. Schirmer O. Studien zur Physiologie and Pathologie der Träneabsonderung und Tränenabfuhr. Graefes Arch Opbthalmol. 1903;56: 197-291.

17. Belmonte C, Acosta MC, Shmelz M, Gallar J. Measurements of corneal sensitivity to mechanical and chemical stimuli with a $\mathrm{CO}_{2}$ esthesiometer. Invest Ophthalmol Vis Sci. 1999;40:513-519.

18. Acosta MC, Peral A, Luna C, Pintor J, Belmonte C, Gallar J. Tear secretion induced by selective stimulation of corneal and conjunctival sensory nerve fibers. Invest Ophthalmol Vis Sci. 2004;45: 2333-2336.

19. Pintor J, Díaz-Rey MA, Torres M, Miras-Portugal MT. Presence of diadenosine polyphosphates $\mathrm{Ap}_{4} \mathrm{~A}$ and $\mathrm{Ap}_{5} \mathrm{~A}$ in rat brain synaptic terminals: $\mathrm{Ca}^{+2}$ dependent release evoked by 4-aminopyridine and veratridine. Neurosci Lett. 1992;136:114-144.

20. Rotllán P, Ramos A, Pintor J, Miras-Portugal MT. Di(1, N6-ethenoadenosine) $5^{\prime}, 5^{\prime \prime \prime}$-P1,P4-tetraphosphate, a fluorescent enzymatically active derivative of $\mathrm{Ap}_{4}$ A. FEBS Lett. 1991;280:371-374.

21. Tsubota K, Hata S, Okusawa Y, Egami F, Ohtsuki T, Nakamori K. Quantitative videographic analysis of blinking in normal subjects and patients with dry eye. Arch Ophthalmol. 1996;114:715-720.

22. Srinivas SP, Mutharasan R, Fleiszig S. Shear-induced ATP release by cultured rabbit corneal epithelial cells. Adv Exp Med Biol. 2002,506:677-685.

23. Taylor AL, Kudlow BA, Marrs KL, Gruenert DC, Guggino WB, Schwiebert EM. Bioluminescence detection of ATP release mechanisms in epithelia. Am J Physiol. 1998;275:C1391-C1406.

24. Wilson PD, Hovater JS, Casey CC, Fortenberry JA, Schwiebert EM. ATP release mechanisms in primary cultures of epithelia derived from the cysts of polycystic kidneys. J Am Soc Nepbrol. 1999;10: 218-229.

25. Mitchell CH, Carre DA, McGlinn AM, Stone R, Civan MM. A release mechanism for stored ATP in ocular ciliary epithelial cells. Proc Natl Acad Sci USA 1998;95:7174-7178.

26. Borst $\mathrm{P}$, Elferink RO. Mammalian ABC transporters in health and disease. Annu Rev Biochem. 2002;71:537-592.

27. Lazarowski ER, Boucher RC, Harden TK. Mechanisms of release of nucleotides and integration of their action as P2X- and P2Y-receptor activating molecules. Mol Pharmacol. 2003;64:785-795.

28. Abraham EH, Prat AG, Gerweck L, et al. The multidrug resistance (mdr1) gene product functions as an ATP channel. Proc Natl Acad Sci USA. 1993;90:312-316.

29. Gomes P, Srinivas SP, Driessche, WV, Vereecke J, Himpens B. ATP release through connexin hemichannels in corneal endothelial cells. Invest Opbthalmol Vis Sci. 2005;46:1208-1218.

30. Records R. The tear film. In: Duane T, Jaeger E, eds. Biomedical Foundations of Opbthalmology. Vol 2. New York: Harper \& Row Publishers Inc.; 1982:1-22.

31. Doanne $M$. Interaction of eyelids and tears in corneal wetting and the dynamics of the normal human eye blink. Am J Opbthalmol. 1980;89:507-516.

32. Acosta MC, Belmonte C, Gallar J. Sensory experience in humans and single-unit activity in cats evoked by polymodal stimulation of the cornea. J Physiol. 2001;534:511-525.

33. Cowlen MS, Zhang VZ, Warnock L, Moyer CF, Peterson WM, Yerxa BR. Localization of ocular $\mathrm{P}_{2} \mathrm{Y}_{2}$ receptor gene expression by in situ hybridization. Exp Eye Res. 2003;77:77-84. 